\title{
Engineering Biomaterials for Enhanced Tissue Regeneration
}

\author{
Rosalyn D. Abbott ${ }^{1}$ - David L. Kaplan ${ }^{1}$
}

Published online: 29 March 2016

(C) Springer International Publishing AG 2016

\begin{abstract}
The formation of artificial organs with tissue engineering techniques is necessary to address the growing disparity between the supply and need for donor organs. For use in tissue engineering regenerative applications, biomaterials should be biocompatible, porous (to allow cellular infiltration, nutrient transport and waste removal), mechanically tunable (to match and maintain the intrinsic mechanical properties of the tissue through the healing process), biodegradable (to allow the tissue to develop as the material degrades), reproducible, easily prepared, and cell/tissue compatible. This review will focus on various biomaterial design considerations and their effect on regenerative outcomes. By adjusting material designs, including pore size and degradation kinetics, in combination with functionalization with cell- and tissue-specific factors, intrinsic properties of tissue constructs can be controlled to enhance remodeling and functional outcomes.
\end{abstract}

Keywords Biomaterial · Tissue regeneration · Tissue engineering $\cdot$ Scaffold $\cdot$ Biodegradable

\section{Introduction}

According to the Organ Procurement and Transplantation Network, there are over 120,000 patients in the USA awaiting

This article is part of the Topical Collection on Artificial Tissues

David L. Kaplan

david.kaplan@tufts.edu

Rosalyn D. Abbott

rosalyn.abbott@tufts.edu

1 Tufts University, 4 Colby St., Medford, MA 02155, USA organ transplantation (as of 15 November 2015). Transplanting a tissue from one location to another within the same patient (an autograft) or from one patient to another patient (an allograft) is effective in many instances for replacing organs with functional outcomes. However, many problems exist with both procedures. Autograft techniques are costly, result in an additional site of injury, increase the risk of infection or hemorrhages, and are limited by anatomically incorrect replacements from other regions of the body [1]. Allografts on the other hand, are often rejected by the immune system (requiring immunosuppressant therapies), and pose risks of infection or transfer of diseases between patients. As an alternative, there has been growing interest in designing replacement organs that use mechanical, physical, and/or biological components to restore or replace function. In particular, tissue engineering historically has involved the concept of isolating cells from a patient, expanding them in vitro, and seeding them onto a biomaterial that could be implanted in vivo in the site of injury [2]. The formation of artificial organs with tissue engineering techniques is necessary to address the growing disparity between the supply and need for donor organs, as well as the limitations associated with autograft and allograft techniques.

For use in tissue engineering regenerative applications, biomaterials should be biocompatible, porous (to allow cellular infiltration, nutrient transport, and waste removal), mechanically tunable (to maintain the intrinsic mechanical properties of the tissue through the healing process), biodegradable (to allow the tissue to develop as the material degrades), reproducible, easily prepared, and cell/tissue compatible. However, optimizing these biomaterial design criteria can result in distinct changes in regenerative potential (Table 1). Therefore, this review will focus on various biomaterial design considerations and their effect on regenerative outcomes (Fig. 1), keeping in mind that tissue-specific considerations [7] will also be required to choose the optimal biomaterial system. 
Table 1 Effects of biomaterial characteristics on regenerative outcomes

\begin{tabular}{|c|c|c|}
\hline Characteristic & Effect on regenerative outcomes & References \\
\hline \multirow[t]{2}{*}{ Adjust the porosity } & $\begin{array}{l}\text { - Alter availability of integrin/ligand binding } \\
\text { sites between cells and the biomaterial } \\
\text { - Change cell/nutrient infiltration }\end{array}$ & \multirow[t]{2}{*}[1,3]{} \\
\hline & - Change mechanical properties & \\
\hline Control degradation & $\begin{array}{l}\text { - Initially support seeded cells (and/or infiltrating cells) } \\
\text { to proliferate and secrete matrix } \\
\text { - Gradually degrade allowing de novo tissue to fill } \\
\text { the empty spaces left by the scaffold }\end{array}$ & {$[4]$} \\
\hline Increase bioactivity & $\begin{array}{l}\text { - Enhance proliferation and/or differentiation } \\
\text { - Foster cell ingress to change remodeling rates }\end{array}$ & {$[5]$} \\
\hline Alter the surface chemistry & - Enhance cell adhesion, proliferation, and/or differentiation & {$[6]$} \\
\hline
\end{tabular}

\section{Biomaterial Sources}

Biomaterials that emulate the native extracellular microenvironment can support or direct the proper cellular phenotypic state. Generally, there are three different approaches for generating the scaffolds required to engineer a tissue (examples, see Table 2): (1) construct scaffolds from natural or synthetic sources $[2,10,11,14],(2)$ decellularize the tissue of interest leaving the extracellular matrix as the scaffolding material [12], or (3) stimulate cells to generate their own matrix
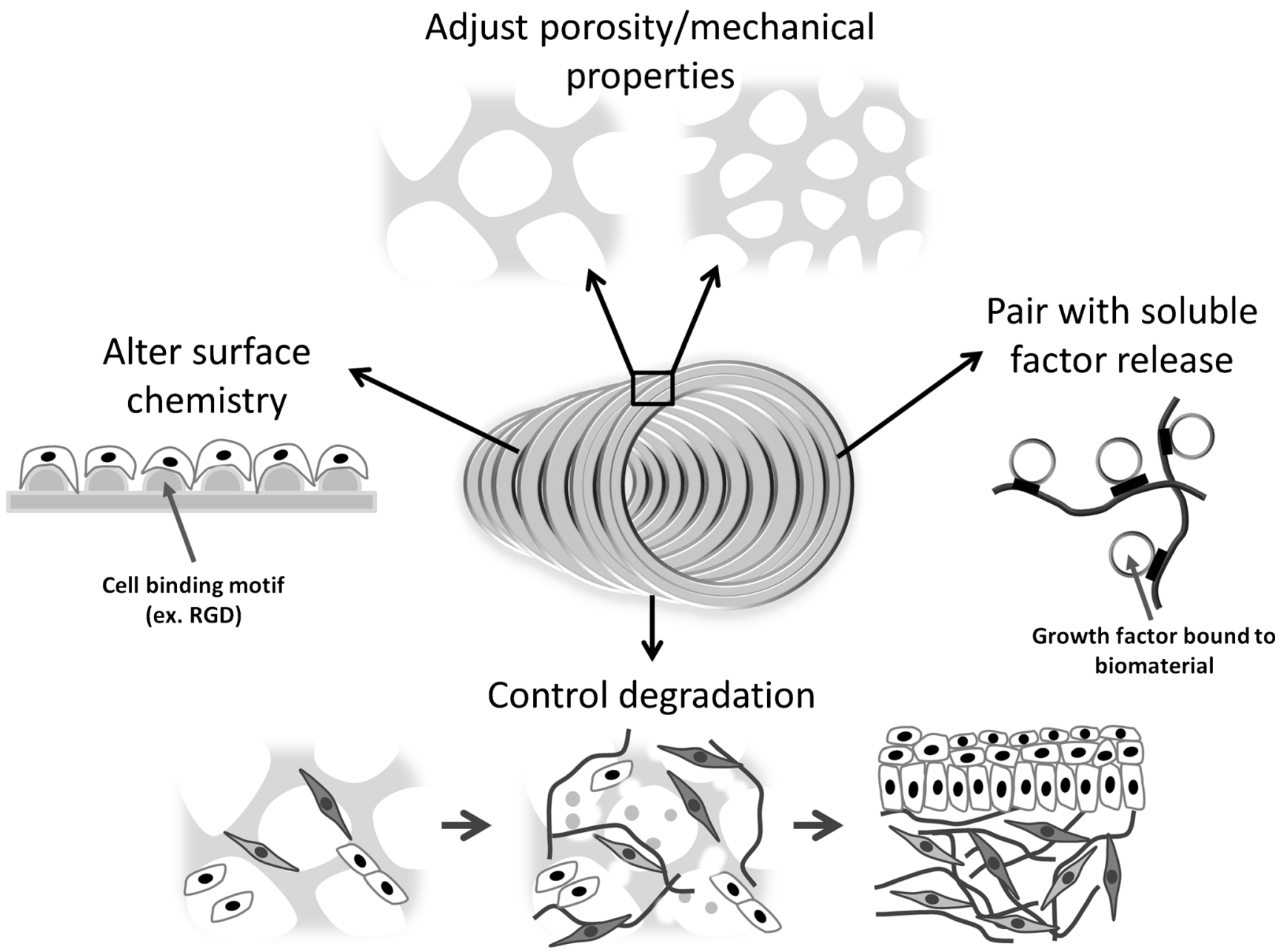

Fig. 1 By adjusting biomaterial designs, including: the porosity and mechanical properties, the surface chemistry, the degradation kinetics, and combining biomaterials with cell- and tissue-specific factors (including soluble factors), intrinsic properties of tissue constructs can be controlled to enhance remodeling and functional outcomes 
Table 2 Examples of biomaterials used in regenerative applications

\begin{tabular}{|c|c|c|}
\hline Biomaterial & Examples & References \\
\hline Synthetic biomaterials & $\begin{array}{l}\text { - Poly(lactic acid) } \\
\text { - Poly-D,L-lactic-glycolic acid co-polymer }\end{array}$ & {$[8,9]$} \\
\hline $\begin{array}{l}\text { Natural biomaterials or genetically } \\
\text { engineered variants }\end{array}$ & $\begin{array}{l}\text { - Collagen } \\
\text { - Silk } \\
\text { - Elastin }\end{array}$ & {$[10,11]$} \\
\hline Decellularized tissue scaffolds & $\begin{array}{l}\text { - Dermis } \\
\text { - Urinary bladder } \\
\text { - Small intestine }\end{array}$ & {$[12]$} \\
\hline Cell-generated scaffolds & $\begin{array}{l}\text { - Collagen } \\
\text { - Elastin }\end{array}$ & {$[13]$} \\
\hline Future biomaterials & $\begin{array}{l}\text { - Patient-specific architecture } \\
\text { - Patient-specific degradation kinetics } \\
\text { - Spatially specific cues within a scaffold } \\
\text { - Temporal control of factors within a } \\
\text { scaffold (release of factors over time) } \\
\text { - Hybrid biomaterials }\end{array}$ & \\
\hline
\end{tabular}

[13]. Each method has advantages and disadvantages that make them more appropriate for certain circumstances based on their inherent biocompatibility, reproducibility, and ease of preparation.

Intuitively, the optimal biomaterial would be the native extracellular matrix of the tissue of interest [7]. Both decellularized tissues and cell-generated materials have specific ligands for cell adhesion, can be degraded and remodeled readily by cells, and have other tissuespecific cues consistent with the native organ, such as signaling epitopes and/or sequestered growth factors [12]. These matrices are naturally biocompatible, however are not easily prepared or reproducible, with relatively low yields of material. Therefore, generating scaffolds from natural or synthetic sources has some advantages over decellularizing tissues or stimulating cells to generate their own matrix. For instance, there is a greater supply of natural and synthetic sources from which to generate the scaffolds. Due to control of processing methods, there is also greater control over the resulting shape and related physical and chemical features of the scaffolds when compared with decelluarized options. Further, the process time required to obtain the material is often faster. Comparing synthetic to biological materials, synthetic materials have minimal lot-to-lot variability, are highly tunable (chemical and mechanical properties), are easier to obtain a purified form, and are generally more reproducible then natural sources [14]. However, natural sources of scaffold materials have advantages such as potentially enhanced biocompatibility, ease of chemical functionalization or sequestering of growth factors, in some cases biological recognition, and susceptibility to cell triggered proteolytic degradation and remodeling [14].

\section{Tuning Mechanics}

The appropriate mechanical characteristics of a biomaterial are highly dependent on the regenerative application. For example, mesenchymal stem cells differentiate into different lineages, such as neurons, myoblasts, and osteoblasts, on progressively stiffer substrates [15]. There are many ways of changing the mechanics of a biomaterial (depending on what type it is). Some common approaches include: crosslinking materials [16], controlling crystallinity during processing [17], and using inorganic reinforcing fillers (to enhance the stiffness of the matrix [18]). Greater precision in scaffold processing such as microfabrication techniques [19] and employing mechanical gradients within the same scaffold system (for soft-to-hard interfaces [20••]) are also being used to more closely mimic in situ architecture.

\section{Porous Structures}

Pores are necessary to allow cells and the nutrients they require to migrate and infiltrate into the bulk of the scaffolds. Pores can be formed by many different processing techniques, including: freeze drying, porogen leaching, gas foaming, microfabrication, and three-dimensional (3D) printers (for a review on processing techniques that result in differences in pore sizes see reference $[21 \bullet \bullet]$ ). While increasing the pore size of a scaffold increases infiltration of cells and improves transport, there is a trade-off with mechanical properties. For example, when biomaterials are implanted in vivo to promote bone ingrowth, higher porosity, and pore sizes result in greater osteo-integration with a reduction of mechanical strength [3]. Larger pore sizes also result in decreased integrin/ligand interactions between cells and the biomaterial [1]. On the other 
hand, decreasing the pore size significantly limits cell migration (surface growth versus bulk infiltration) and can lead to cellular encapsulation of the scaffold [1]. While larger pores and enhanced porosity increases nutrient transfer, this can also be achieved by adding channels to scaffolds [22-26]. In some tissue engineering constructs, these channels are lined with endothelial cells [27-30] to recreate the network of vasculature that provides nutrients in vivo. It is important to consider the dynamic nature of endothelial networks, however, which is dependent on the type of mural cells and the mechanical and bioactive properties of the matrix [31]. In addition, the interconnectivity of pores and adding channels to the materials, affects the mechanical properties where randomly oriented defects from both processing techniques soften the bulk properties of tissue constructs resulting in problems generating structurally robust tissues (compared with more ordered arrangements). Therefore, careful consideration should be taken when choosing the porous (and/or channel) structure of biomaterials.

\section{Controlled Degradation}

One of the goals of tissue engineering is to design the degradation and remodeling kinetics of a biomaterial to: (1) initially support seeded cells (and/or infiltrating cells) to proliferate and secrete matrix, and (2) gradually degrade allowing de novo tissue to fill the empty spaces left by the scaffold. In this way, paired scaffold degradation and tissue remodeling can maintain the intrinsic mechanical properties through the healing process. Unfortunately, each tissue requires unique degradation/resorption kinetics based on differences in matrix properties, cell types, and bioactivity of other factors in the extracellular matrix. An added confounder is that the regenerative capacity of patients varies, for example, children exhibit higher regenerative capacity and will likely require a faster degrading scaffold than adults that have a slower healing capacity [4]. Other variables aside from age include patient nutritional status, disease state, lifestyle, sex, and other related factors.

Materials can be biodegradable, bioresorbable, bioerodible, or bioabsorbable [32]. Biodegradable materials break down due to macromolecular degradation to byproducts that can move away from the implantation site but are not necessarily removed from the body $[32,33]$. In contrast, bioresorbable materials break down due to bulk degradation and are eliminated through natural pathways (such as metabolism by cells) with no residual side effects $[32,33]$. This is ideal in some circumstances, as complete removal of the biomaterial eliminates any remaining foreign body reaction. Alternatively, bioerodible polymers degrade and resorb only at the surface $[32,33]$. Finally, bioabsorbable materials dissolve in body fluids without a decrease in molecular mass (a bioabsorbable material is bioresorbable if the byproducts are excreted). All classifications result in different regenerative outcomes, and are highly dependent on tissue-specific requirements.

An important consideration is whether the degradation byproducts will have toxic effects on cells and the surrounding tissue. To address degradation and remodeling concerns, it is important to consider the source of the material. Many synthetic polymers are biodegradable, for example, the copolymer poly-D,L-lactic-glycolic acid (PLGA). However, the acidic environment generated by the degradation of PLGA is detrimental to cell mobilization and angiogenesis [8]. Proteins derived from the extracellular matrix, on the other hand, are naturally susceptible to proteolytic degradation and remodeling by cells. The byproducts of degradation are less likely to have toxic effects; however, the byproducts may cause downstream impact. For instance, byproducts of collagen degradation control cell migration of vascular smooth muscle cells which are responsible for de novo synthesis of specific extracellular matrix components (including restenosis after balloon angioplasty) [34].

An interesting approach to controlling degradation in polymers is with biological triggers. In this approach, labile chemical groups are conjugated into the backbone of polymers that can be degraded by physical or chemical signals, such as nearinfrared light [35], ultraviolet light [36•], ultrasound [37, 38], redox [4], various enzymes [39, 40], and $\mathrm{pH}$ changes [41]. As an example, a peptide-conjugated polymer was generated, which underwent degradation when it was exposed to collagenase [39]. When designing these systems, the physical or chemical cue must be carefully designed to avoid off target effects. For instance, some enzymes, such as collagenase, can cause further damage to the surrounding tissue. Targeting local cell types that release the enzyme of interest (for instance MMPs [40]), or directly injecting biological triggers into the implantation site [4], are approaches that can be explored to reduce injury to the surrounding tissue.

\section{Release of Soluble Factors}

To improve biological activity, controlled biomaterial degradation can be paired with the release of soluble factors to enhance regenerative outcomes. Drugs have been incorporated in many biomaterial scaffolds, for example, to promote angiogenesis in ischemic tissues [42]. Another important area of research is the controlled delivery of growth factors, which are important mediators of cellular signaling, migration, proliferation, differentiation, and maturation [2]. To incorporate both drugs and growth factors in regenerative approaches, local and temporal control is required to improve clinical outcomes [43]. To accomplish this task, soluble factors have been delivered from particles (at the micro- and nano- scale), from scaffolds, and also encapsulated to release as the biomaterials are degraded [5]. Binding of growth factors and drugs to 
biomaterials can occur through covalent [44] or ionic bonds [45] and depends on the properties of the soluble factors and the biomaterial. Encapsulating growth factors or drugs helps to control the release profile and can protect the unstable bioactive molecules from degradation (and loss of activity). However, covalent/ionic bonds and encapsulation alone often lead to an initial burst release of soluble factors. Many approaches aimed at accomplishing sustained release and zero-order release have been proposed to address the burst issue (for reviews see: [46, 47]). For example, in one system [48], bone morphogenetic protein 2 was encapsulated in a nano-sphere which was encapsulated in a micro-sphere scaffolding system to enhance bone regeneration. The hierarchical structure minimized the initial burst from the nano-sphere by confining it within the scaffolding system of the micro-sphere. Other biomaterial approaches control temporal timing of factors to release different factors over time. For example, epidermal growth factor at early time points and mitomycin $\mathrm{C}$ at later time points [49].

\section{Other Surface Chemistry Considerations}

When cellular binding sites are not present on the material, cell adhesion ligands such as proteins, peptides, and oligosaccharides can be added to enhance cell adhesion. Coating with extracellular matrix proteins which are specific for certain tissues is commonly used to enhance adhesion, differentiation, and/or proliferation of certain cell types. For instance, fibronectin [50], collagen [51], and laminin [52], some of the most common protein coatings used, have all been recently used to enhance tissue-specific regenerative outcomes for periodontal ligament, smooth muscle, and tracheal epithelial tissue, respectively.

Coating with proteins has several drawbacks. Proteins must be isolated and purified from an organism, making large-scale production challenging, or they can be generated by recombinant DNA methods, which adds cost and purification challenges. They may also elicit immune responses and can be proteolytically degraded [6]. Decorating the materials with immmobilized peptides for cell recognition motifs, such as RGD (arginine (R), glycine (G), aspartic acid (D)) therefore can be used to counteract the issues associated with protein coatings since they are easily characterized, cost effective, exhibit higher stability, and can be packed at a higher density on the surface of biomaterials [6]. However, immobilized cell recognition motifs may have lower cell adhesion activity and are specific to only one cell adhesion motif (whereas matrix proteins have many) [53].

\section{Future Considerations for Biomaterials Aiding Tissue Regeneration}

Creative approaches to adjust material designs, including pore size and degradation kinetics, in combination with functionalization with cell- and tissue-specific factors will result in enhanced tissue remodeling and functional outcomes. Greater precision in scaffold processing is required to provide control over the structure of biomaterials to recreate tissuespecific features. Advances in 3D printing and nanotechnology development will likely be paramount for this precision. In particular, patient- and tissue-specific architectures and degradation kinetics will be a goal. In this way, scaffolds can be designed to match the geometry of the patient-specific defect and account for location, age, or disease state for degradation properties. In addition, it will be important to modulate specific cues spatially and temporally within a scaffold, for instance by coating specific regions with different extracellular matrix proteins or by pairing degradation with the release of specific soluble factors. This approach will be especially important in cases where multiple cell types or tissues exist within one organ. Likewise, creating gradients and accounting for interfaces (i.e., between soft and hard tissues) with different biomaterial processing techniques will be crucial. Hybrid materials that combine the advantages of different materials will also be essential in many applications. As an example, extracellular matrix/fibrin scaffolds were generated, which combined the highly angiogenic properties of fibrin with cardiac extracellular matrix to promote both vascular and cardiac differentiation within the same scaffold [54•]. This hybrid system also resulted in varying levels of mechanics, which will be an important consideration for biomaterials moving forward. As these new areas of biomaterial development are explored, standardization, good manufacturing practices, and costeffective methods will be essential for translation toward clinical utility.

\section{Conclusions}

Emulating the extracellular microenvironment is essential for establishing proper cellular phenotypes required for tissue regeneration. Careful consideration of biomaterial choices in tissue engineering can improve regenerative outcomes. For instance, by making adjustments to the material design, including pore size and degradation kinetics, intrinsic properties of tissue constructs can be controlled to enhance remodeling and functional outcomes. Additionally, the extracellular matrix is a repository for a variety of growth factors and other signaling ligands, which can be incorporated in biomaterial approaches to improve regenerative outcomes. 
Acknowledgments We thank the NIH (P41 EB002520, R01 EY020856, R01 DE017207, R01 EB014283) for support of this work.

\section{Compliance with Ethical Standards}

Conflict of Interest Rosalyn D. Abbott and David L. Kaplan declare that they have no conflict of interest.

Human and Animal Rights and Informed Consent This article does not contain any studies with human or animal subjects performed by any of the authors.

\section{References}

Papers of particular interest, published recently, have been highlighted as:

- Of importance

•- Of major importance

1. Murphy CM, O'Brien FJ. Understanding the effect of mean pore size on cell activity in collagen-glycosaminoglycan scaffolds. Cell Adhes Migr. 2010;4(3):377-81.

2. Langer R, Vacanti JP. Tissue engineering. Science. 1993;260(5110):920-6.

3. Karageorgiou V, Kaplan D. Porosity of 3D biomaterial scaffolds and osteogenesis. Biomaterials. 2005;26(27):5474-91.

4. $\mathrm{Xu} \mathrm{C}$ et al. Triggerable degradation of polyurethanes for tissue engineering applications. ACS Appl Mater Interfaces. 2015;7(36): 20377-88.

5. Chen RR, Mooney DJ. Polymeric growth factor delivery strategies for tissue engineering. Pharm Res. 2003;20(8):1103-12.

6. Hersel U, Dahmen C, Kessler H. RGD modified polymers: biomaterials for stimulated cell adhesion and beyond. Biomaterials. 2003;24(24):4385-415.

7. Chan BP, Leong KW. Scaffolding in tissue engineering: general approaches and tissue-specific considerations. Eur Spine J. 2008;17 Suppl 4:467-79.

8. Sung HJ et al. The effect of scaffold degradation rate on threedimensional cell growth and angiogenesis. Biomaterials. 2004;25(26):5735-42.

9. Lasprilla AJR et al. Poly-lactic acid synthesis for application in biomedical devices - a review. Biotechnol Adv. 2012;30(1):321-8.

10. Kundu B et al. Silk proteins for biomedical applications: bioengineering perspectives. Prog Polym Sci. 2014;39(2):251-67.

11. Langer R, Tirrell DA. Designing materials for biology and medicine. Nature. 2004;428(6982):487-92.

12. Brown BN, Badylak SF. Extracellular matrix as an inductive scaffold for functional tissue reconstruction. Transl Res. 2014;163(4): 268-85.

13. Schella JY et al. Harnessing cellular-derived forces in selfassembled microtissues to control the synthesis and alignment of ECM. Biomaterials. 2015;77:120-9.

14. Lutolf MP, Hubbell JA. Synthetic biomaterials as instructive extracellular microenvironments for morphogenesis in tissue engineering. Nat Biotechnol. 2005;23(1):47-55.

15. Engler AJ et al. Matrix elasticity directs stem cell lineage specification. Cell. 2006;126(4):677-89.

16. Weadock K, Olson RM, Silver FH. Evaluation of collagen crosslinking techniques. Biomater Med Devices Artif Organs. 1983;11(4):293-318.
17. Xiao $\mathrm{S}$ et al. Mechanical response of silk crystalline units from force-distribution analysis. Biophys J. 2009;96(10):3997-4005.

18. $\mathrm{Xu} \mathrm{HH}$ et al. Effects of incorporating nanosized calcium phosphate particles on properties of whisker-reinforced dental composites. J Biomed Mater Res B Appl Biomater. 2007;81(1):116-25.

19. Vozzi G, Ahluwalia A. Microfabrication for tissue engineering: rethinking the cells-on-a scaffold approach. J Mater Chem. 2007;17(13):1248-54.

20.• Seidi A et al. Gradient biomaterials for soft-to-hard interface tissue engineering. Acta Biomater. 2011;7(4):1441-51. This review focusess on fabrication techniques for generating gradient biomaterials. It explores specific experimental examples of gradient tissue engineering from soft tissues (e.g. cartilage) to hard tissues (e.g. bone).

21.• Loh QL, Choong C. Three-dimensional scaffolds for tissue engineering applications: role of porosity and pore size. Tissue Eng B Rev. 2013;19(6):485-502. This review focuses on fabrication techniques for generating different pore sizes, porosity, and graded porous networks. It also evaluates how scaffold pore size and porosity directs cellular responses and the resulting mechanical properties.

22. Wray LS et al. A silk-based scaffold platform with tunable architecture for engineering critically-sized tissue constructs. Biomaterials. 2012;33(36):9214-24.

23. Radisic $\mathrm{M}$ et al. Biomimetic approach to cardiac tissue engineering: oxygen carriers and channeled scaffolds. Tissue Eng. 2006;12(8): 2077-91.

24. Nazhat SN et al. Controlled microchannelling in dense collagen scaffolds by soluble phosphate glass fibers. Biomacromolecules. 2007;8(2):543-51.

25. Bagnaninchi PO et al. Chitosan microchannel scaffolds for tendon tissue engineering characterized using optical coherence tomography. Tissue Eng. 2007;13(2):323-31.

26. Moore MJ et al. Multiple-channel scaffolds to promote spinal cord axon regeneration. Biomaterials. 2006;27(3):419-29.

27. Zheng $\mathrm{Y}$ et al. In vitro microvessels for the study of angiogenesis and thrombosis. Proc Natl Acad Sci U S A. 2012;109(24):9342-7.

28. Morgan JP et al. Formation of microvascular networks in vitro. Nat Protoc. 2013;8(9):1820-36.

29. Schimek K et al. Integrating biological vasculature into a multiorgan-chip microsystem. Lab Chip. 2013;13(18):3588-98.

30. Tiruvannamalai-Annamalai R, Armant DR, Matthew HW. A glycosaminoglycan based, modular tissue scaffold system for rapid assembly of perfusable, high cell density, engineered tissues. PLoS One. 2014;9(1):e84287.

31. Chwalek K et al. Glycosaminoglycan-based hydrogels to modulate heterocellular communication in in vitro angiogenesis models. Sci Rep. 2014;4:4414.

32. Hutmacher DW. Scaffolds in tissue engineering bone and cartilage. Biomaterials. 2000;21(24):2529-43.

33. Vert $\mathrm{M}$ et al. Bioresorbability and biocompatibility of aliphatic polyesters. J Mater Sci Mater Med. 1992;3(6):432-46.

34. Stringa $\mathrm{E}$ et al. Collagen degradation and platelet-derived growth factor stimulate the migration of vascular smooth muscle cells. J Cell Sci. 2000;113(11):2055-64.

35. Wu G et al. Remotely triggered liposome release by near-infrared light absorption via hollow gold nanoshells. J Am Chem Soc. 2008;130(26):8175-7.

36. Nazemi A, Schon TB, Gillies ER. Synthesis and degradation of backbone photodegradable polyester dendrimers. Org Lett. 2013;15(8):1830-3. This article demonstrates a well-defined, fully photodegradable material suited for triggerable biological degradation.

37. Epstein-Barash $\mathrm{H}$ et al. A microcomposite hydrogel for repeated on-demand ultrasound-triggered drug delivery. Biomaterials. 2010;31(19):5208-17. 
38. Kost J, Leong K, Langer R. Ultrasound-enhanced polymer degradation and release of incorporated substances. Proc Natl Acad Sci U S A. 1989;86(20):7663-6.

39. Fu HL et al. Collagenase-labile polyurethane urea synthesis and processing into hollow fiber membranes. Biomacromolecules. 2014;15(8):2924-32.

40. Lutolf MP et al. Synthetic matrix metalloproteinase-sensitive hydrogels for the conduction of tissue regeneration: engineering cell-invasion characteristics. Proc Natl Acad Sci U S A. 2003;100(9):5413-8.

41. El-Sayed ME, Hoffman AS, Stayton PS. Rational design of composition and activity correlations for $\mathrm{pH}$-responsive and glutathione-reactive polymer therapeutics. J Control Release. 2005;104(2):417-27.

42. Rufaihah AJ, Seliktar D. Hydrogels for therapeutic cardiovascular angiogenesis. Adv Drug Deliv Rev. 2015;96:31-9.

43. Babensee JE, McIntire LV, Mikos AG. Growth factor delivery for tissue engineering. Pharm Res. 2000;17(5):497-504.

44. Yang $\mathrm{Y}$ et al. Nerve conduits based on immobilization of nerve growth factor onto modified chitosan by using genipin as a crosslinking agent. Eur J Pharm Biopharm. 2011;79(3):519-25.

45. Coburn JM, Na E, Kaplan DL. Modulation of vincristine and doxorubicin binding and release from silk films. J Control Release. 2015;220(Pt A):229-38

46. Pritchard EM, Kaplan DL. Silk fibroin biomaterials for controlled release drug delivery. Expert Opin Drug Deliv. 2011;8(6):797-811.
47. Coburn JM, Kaplan DL. Engineering biomaterial-drug conjugates for local and sustained chemotherapeutic delivery. Bioconjug Chem. 2015;26(7):1212-23.

48. Ma $\mathrm{C}$ et al. Hierarchical nanofibrous microspheres with controlled growth factor delivery for bone regeneration. Adv Healthc Mater. 2015;4:2699-708.

49. Zhu J et al. A tubular gelatin scaffold capable of the time-dependent controlled release of epidermal growth factor and mitomycin C. Colloids Surf B: Biointerfaces. 2015;135:416-24.

50. $\mathrm{Li} \mathrm{H}$ et al. Spray-painted human fibronectin coating as an effective strategy to enhance graft ligamentization of a polyethylene terephthalate artificial ligament. Biotechnol Lett. 2014;36(5):1079-88.

51. Sharifiaghdas $\mathrm{F}$ et al. Comparing supportive properties of poly lactic-co-glycolic acid (PLGA), PLGA/collagen and human amniotic membrane for human urothelial and smooth muscle cells engineering. Urol J. 2014;11(3):1620-8.

52. Lee DY et al. Synergistic effect of laminin and mesenchymal stem cells on tracheal mucosal regeneration. Biomaterials. 2015;44:134 42 .

53. Ruoslahti E. RGD and other recognition sequences for integrins Annu Rev Cell Dev Biol. 1996;12:697-715.

54. Williams $\mathrm{C}$ et al. Cardiac extracellular matrix-fibrin hybrid scaffolds with tunable properties for cardiovascular tissue engineering. Acta Biomater. 2015;14:84-95. This article demonstrates an example of a hybrid scaffold. An extracellular matrix / fibrin scaffold was generated that explores the effects of exploiting both composition and mechanical properties to control the differentiation of vascular and cardiac components. 